Physics Notes

Note 18

February 2007

\title{
Path Integrals in Electromagnetics
}

\author{
Carl E. Baum \\ University of New Mexico \\ Department of Electrical and Computer Engineering \\ Albuquerque New Mexico 87131
}

\begin{abstract}
In searching for various representations of the electromagnetic fields, some recent interest has been placed on the use of path integrals. These in some sense propagate fields from previous times to sometime of interest by all possible spatial paths appropriate to the time difference to the present. This paper reviews some standard formulations and compares them to the path-integral formulation to give an explicit interpretation for electromagnetics.
\end{abstract}

This work was sponsored in part by the Air Force Office of Scientific Research. 
1. Introduction

In representing the solution to electromagnetic problems (e.g., radiation, scattering, propagation) in various media, there are various possible approaches. One can solve differential equations appropriate to special geometries (e.g., waveguides, cavities). One can formulate integral equations over antennas and scatterers using appropriate Green functions, usually of the free-space variety. One can discretize the Maxwell equations in frequency and time domains for a numerical (computer) solution to geometries and media with intractable analytic solutions. Symmetries can also be used to reduce the solution difficulty [16]. The above can be elaborated in various ways using various mathematical techniques [3-5, 7, 12, 15].

Another potential approach involves the path integral introduced by Feynman [6, 11]. This has been introduced into electromagnetics (e.g., [8, 9]). Intuitively, the path integral represents the electromagnetic fields at some point in space by integrals over other portions of space along all possible paths between each coordinate, $\overrightarrow{r^{\prime}}$, to the position, $\vec{r}$, of interest. Of course, causality can be invoked to limit the paths of integration to previous times, and to regions of space where there are nonzero fields and sources within a transit time on a path from $\overrightarrow{r^{\prime}}$ to $\vec{r}$.

Given that there are established valid representations of electromagnetic fields, it is the purpose of this paper to clarify the path integral representation in terms of these other representations. 
2. Green Function Representation for Uniform Isotropic Media

2.1 Spatially dependent constitutive parameters

Start with the Maxwell equations and constitutive relations as

$$
\begin{aligned}
& \nabla \times \vec{E}(\vec{r}, t)=-\frac{\partial \vec{B}(\vec{r}, t)}{\partial t}-\vec{J}_{h}(\vec{r}, t) \\
& \nabla \times \vec{H}(\vec{r}, t)=-\frac{\partial \vec{D}(\vec{r}, t)}{\partial t}+\vec{J}_{e}(\vec{r}, t) \\
& \overleftrightarrow{D}(\vec{r}, t)=\overleftrightarrow{\varepsilon}(\vec{r}) \cdot \vec{E}(\vec{r}, t) \\
& \overleftarrow{B}(\vec{r}, t)=\overleftrightarrow{\mu}(\vec{r}) \cdot \vec{H}(\vec{r}, t)
\end{aligned}
$$

Here we have taken $\stackrel{\leftrightarrow}{\varepsilon}$ and $\overleftrightarrow{\mu}$ as functions of space, but not of frequency. The medium conductivity (if any) can be handled by the transformation

$$
\frac{\partial \vec{D}(\vec{r}, t)}{\partial t}=\overleftrightarrow{\sigma}(\vec{r}) \cdot \vec{E}(\vec{r}, t)+\overleftrightarrow{\varepsilon}(\vec{r}) \cdot \frac{\partial \vec{E}(\vec{r}, t)}{\partial t}
$$

One can include $\overleftrightarrow{\sigma}$ in a frequency-dependent $\stackrel{\leftrightarrow}{\varepsilon}$. It can also be included in the source term $\vec{J} e$. For symmetry one could also include a magnetic conductivity, or lump it in $\vec{J}_{h}$. This leaves $\vec{J}_{e}$ and $\vec{J}_{h}$ as source terms.

\subsection{Uniform isotropic media}

Specialize now to the case of uniform isotropic media given by

$$
\begin{aligned}
& \overleftrightarrow{\varepsilon}(\vec{r})=\varepsilon \overleftrightarrow{1} \quad, \quad \overleftrightarrow{\mu}(\vec{r})=\mu \overleftrightarrow{1} \\
& \stackrel{\leftrightarrow}{1}=\overrightarrow{1}_{x} \overrightarrow{1}_{x}+\overrightarrow{1}_{y} \overrightarrow{1}_{y}+\overrightarrow{1}_{z} \overrightarrow{1}_{z}=\left(\begin{array}{lll}
1 & 0 & 0 \\
0 & 1 & 0 \\
0 & 0 & 1
\end{array}\right) \text { \# identity 3 x 3 dyadic }
\end{aligned}
$$

Now the Maxwell equations take the form 


$$
\begin{aligned}
& \nabla \times \vec{E}(\vec{r}, t)=-\mu \frac{\partial \vec{H}(\vec{r}, t)}{\partial t}-\vec{J}_{h}(\vec{r}, t) \\
& \nabla \times \vec{H}(\vec{r}, t)=\varepsilon \frac{\partial \vec{E}(\vec{r}, t)}{\partial t}-\vec{J}_{e}(\vec{r}, t)
\end{aligned}
$$

This can be cast in $6 \times 6$ matrix form as

$$
\begin{aligned}
\frac{\partial}{\partial t}\left(\begin{array}{c}
\vec{E}(\vec{r}, t) \\
\vec{H}(\vec{r}, t)
\end{array}\right) & =\left(\begin{array}{cc}
0 & Z^{-1} \varepsilon^{-1} \nabla \times \\
-Z^{-1} \mu^{-1} \nabla \times & 0
\end{array}\right)=\left(\begin{array}{c}
\vec{E}(\vec{r}, t) \\
\vec{Z}(\vec{r}, t)
\end{array}\right)-\left(\begin{array}{c}
\vec{J}_{e}(\vec{r}, t) \\
\vec{J}_{h}(\vec{r}, t)
\end{array}\right) \\
Z & \equiv \text { convenient scaling constant with dimensions of impedance }
\end{aligned}
$$

If now we choose

$$
Z \equiv\left[\frac{\mu}{\varepsilon}\right]^{1 / 2} \equiv \text { wave impedance of medium }
$$

the Maxwell equations can be reduced to a single $3 \times 3$ matrix equation for the combined field with

$$
\begin{aligned}
& \vec{E}_{q}(\vec{r}, t)=\vec{E}(\vec{r}, t)+q j Z \vec{H}(\vec{r}, t) \equiv \text { combined field } \\
& \vec{J}_{q}(\vec{r}, t)=\vec{J}_{e}(\vec{r}, t)+\frac{q j}{Z} \vec{J}_{h}(\vec{r}, t) \equiv \text { combined current density } \\
& q= \pm 1 \equiv \text { separation index }
\end{aligned}
$$

giving

$$
\begin{aligned}
& {\left[\nabla \times \frac{-q j}{c}\right] \vec{E}_{q}(\vec{r}, t)=q j Z \vec{J}_{q}(\vec{r}, t)} \\
& c \equiv[\mu \varepsilon]^{-1 / 2} \equiv \text { speed of light in medium }
\end{aligned}
$$

for the combined Maxwell equation.

\subsection{Green functions}

Now we can exhibit appropriate well-known Green functions for uniform isotropic media. First we have the scalar form 


$$
\begin{aligned}
\tilde{G}_{0}\left(\vec{r}-\overrightarrow{r^{\prime}}, s\right)=\frac{e^{-\gamma\left|\vec{r}-\vec{r}^{\prime}\right|}}{4 \pi\left\|\vec{r}-\vec{r}^{\prime}\right\|} \\
\gamma \equiv \frac{s}{c} \equiv \text { propagation constant } \\
s=\Omega+j \omega \equiv \text { Laplace-tranform variable or complex frequency } \\
\sim \equiv \text { Laplace-transform (two sided) over time, } t
\end{aligned}
$$

in frequency-domain form. In this form, $Z$ and $c$ can be frequency dependent. In turn, this can be used to calculate vector and scalar potentials (Lorentz gauge) as

$$
\begin{aligned}
& \tilde{\vec{A}}_{e}(\vec{r}, s)=\int_{V} \tilde{G}_{0}\left(\vec{r}-\vec{r}^{\prime}, s\right) \vec{J}_{e}\left(\vec{r}^{\prime}, s\right) d V^{\prime} \\
& \tilde{\vec{\Phi}}_{e}(\vec{r}, s)=\int_{V} \tilde{G}_{0}\left(\vec{r}-\vec{r}^{\prime}, s\right) \tilde{\rho}_{e}\left(\vec{r}^{\prime}, s\right) d V^{\prime} \\
& \tilde{\vec{A}}_{h}(\vec{r}, s)=\int_{V} \tilde{G}_{0}\left(\vec{r}-\vec{r}^{\prime}, s\right) \vec{J}_{h}\left(\vec{r}^{\prime}, s\right) d V^{\prime} \\
& \tilde{\Phi}_{e}(\vec{r}, s)=\int_{V} \tilde{G}_{0}\left(\vec{r}-\vec{r}^{\prime}, s\right) \tilde{\rho}_{h}\left(\vec{r}^{\prime}, s\right) d V^{\prime} \\
& 0=\nabla \cdot \tilde{\vec{J}}_{e}(\vec{r}, s)+s \tilde{\rho}_{e}(\vec{r}, s) \text { (electric current continuity) } \\
& \quad \tilde{\overrightarrow{\vec{J}}}_{h}(\vec{r}, s)+s \tilde{\rho}_{h}(\vec{r}, s) \text { (magnetic current continuity) }
\end{aligned}
$$

In time domain we have

$$
G_{0}\left(\vec{r}-\overrightarrow{r^{\prime}}, t-t^{\prime}\right)=\frac{1}{4 \pi\left|\vec{r}-\overrightarrow{r^{\prime}}\right|} \delta\left(t-t^{\prime}-\frac{\left|\vec{r}-\overrightarrow{r^{\prime}}\right|}{c}\right)
$$

The potentials are easily given time-domain form by noting that convolution with a delta function is merely a time shift (delay, enforcing causality).

The fields are now calculable from

$$
\begin{aligned}
& \vec{E}(\vec{r}, t)=-\nabla \Phi_{e}(\vec{r}, t)-\frac{\partial}{\partial t} \vec{A}_{e}(\vec{r}, t)-\frac{1}{\varepsilon} \nabla \times \vec{A}_{h}(\vec{r}, t) \\
& \vec{H}(\vec{r}, t)=\frac{1}{\mu} \nabla \times \vec{A}_{e}(\vec{r}, t)-\nabla \Phi_{h}(\vec{r}, t)-\frac{\partial}{\partial t} \vec{A}_{h}(\vec{r}, t)
\end{aligned}
$$


In combined form we have

$$
\begin{aligned}
& \vec{A}_{q}(\vec{r}, t)=\vec{A}_{e}(\vec{r}, t)+j q Z \vec{A}_{h}(\vec{r}, t) \\
& \Phi_{q}(\vec{r}, t)=\Phi_{e}(\vec{r}, t)+j q Z \Phi_{h}(\vec{r}, t) \\
& \vec{E}_{q}(\vec{r}, t)=-\nabla \Phi_{q}(\vec{r}, t)-\frac{\partial}{\partial t} \vec{A}_{q}(\vec{r}, t)+j q c \nabla \times \vec{A}_{q}(\vec{r}, t)
\end{aligned}
$$

Additional details can be found in [16].

\subsection{Dyadic Green functions}

One can go directly from the currents to the fields via the dyadic Green functions [2, 7]. These are constructed as

$$
\begin{aligned}
\nabla \tilde{G}_{0}\left(\vec{r}-\vec{r}^{\prime}, s\right)= & \overrightarrow{1}_{R} \frac{\gamma^{2}}{4 \pi}\left[-\zeta^{-2}-\zeta^{-1}\right] e^{-\zeta} \\
\stackrel{\leftrightarrow}{G}_{0}\left(\vec{r}-\vec{r}^{\prime} ; s\right)= & \frac{\gamma}{4 \pi}\left[\left[-2 \zeta^{-3}-2 \zeta^{-2}\right] \overrightarrow{1}_{R} \overrightarrow{1}_{R}+\left[\zeta^{-3}+\zeta^{-2}+\zeta^{-1}\right]\left[\stackrel{\leftrightarrow}{1}-\overrightarrow{1}_{R} \overrightarrow{1}_{R}\right]\right] e^{-\zeta} \\
& +\frac{1}{3 \gamma} \delta\left(\vec{r}-\vec{r}^{\prime}\right) \stackrel{\leftrightarrow}{1}
\end{aligned}
$$

where the last term corresponds to a principal-value integral using a spherical small volume centered on $\vec{r}-\overrightarrow{r^{\prime}}=0$. Here we have

$$
\begin{aligned}
& R=\left|\vec{r}-\overrightarrow{r^{\prime}}\right|, \quad \overrightarrow{1}_{R}=\frac{\vec{r}-\overrightarrow{r^{\prime}}}{\left|\vec{r}-\overrightarrow{r^{\prime}}\right|} \\
& \zeta=\gamma R
\end{aligned}
$$

We also have

$$
\nabla \tilde{G}_{0}\left(\vec{r}-\vec{r}^{\prime}, s\right) \times=\nabla \times \stackrel{\leftrightarrow}{G}_{0}\left(\vec{r}-\vec{r}^{\prime}, s\right) \bullet
$$

In combined form we have 


$$
\stackrel{\leftrightarrow}{G}_{0_{q}}\left(\vec{r}-\vec{r}^{\prime}, s\right)=\left[\stackrel{\leftrightarrow}{1} \cdot-\frac{q j}{\gamma} \nabla \times\right] \ddot{\leftrightarrow}_{0}\left(\vec{r}, \overrightarrow{r^{\prime}} ; s\right)
$$

The fields are then found from

$$
\begin{aligned}
& \tilde{\vec{E}}_{q}(\vec{r}, s)=-s \mu \int_{V} \stackrel{\leftrightarrow}{G}_{0_{q}}\left(\vec{r}-\vec{r}^{\prime}, s\right) \cdot \dot{\vec{J}}_{q}\left(\vec{r}^{\prime}, s\right) d V^{\prime} \\
& \tilde{\vec{E}}(\vec{r}, s)=-s \mu \int_{V} \stackrel{\leftrightarrow}{G}_{0}\left(\vec{r}-\vec{r}^{\prime}, s\right) \cdot \tilde{\vec{J}} e\left(\vec{r}^{\prime}, s\right) d V^{\prime} \\
& -\int_{V} \nabla \tilde{G}_{0}\left(\vec{r}-\vec{r}^{\prime}, s\right) \times \tilde{\vec{J}}_{h}\left(\vec{r}^{\prime}, s\right) d V^{\prime} \\
& \tilde{\vec{H}}(\vec{r}, s)=\int_{V} \nabla \stackrel{\leftrightarrow}{G}_{0}\left(\vec{r}-\vec{r}^{\prime}, s\right) \times \tilde{\vec{J}} e\left(\vec{r}^{\prime}, s\right) d V^{\prime} \\
& -s \varepsilon \int_{V} \stackrel{\leftrightarrow}{G}_{0}\left(\vec{r}-\vec{r}^{\prime}, s\right) \cdot \dot{\vec{J}}_{h}\left(\vec{r}^{\prime}, s\right) d V^{\prime}
\end{aligned}
$$

Like (2.11) the dyadic Green functions can also be placed in time domain to give a convolution integral over the currents in (2.18). However, noting that $s^{-1}$ corresponds to a temporal integral (in addition to the convolution and time differentiation) the resulting expressions, while straightforward, are more elaborate. 
3. Maxwell Equations in Inhomogeneous, Anisotropic Media

The Maxwell Equations (2.1) now become

$$
\begin{aligned}
& \nabla \times \vec{E}(\vec{r}, t)=-\overleftrightarrow{\mu}(\vec{r}) \cdot \frac{\partial}{\partial t} \vec{H}(\vec{r}, t)-\vec{J}_{h}(\vec{r}, t) \\
& \nabla \times \vec{H}(\vec{r}, t)=\overleftrightarrow{\varepsilon}(\vec{r}) \cdot \frac{\partial}{\partial t} \vec{E}(\vec{r}, t)+\vec{J}_{e}(\vec{r}, t)
\end{aligned}
$$

This can be cast into a single 6 × 6 matrix equation as

$$
\begin{aligned}
& \frac{\partial}{\partial t}\left(\begin{array}{c}
\vec{E}(\vec{r}, t) \\
\vec{H}(\vec{r}, t)
\end{array}\right)=\left(\begin{array}{cc}
\overleftrightarrow{\leftrightarrow} & Z^{-1} \stackrel{\leftrightarrow}{\varepsilon}^{-1}(\vec{r}) \cdot \nabla \times \\
0 & \overleftrightarrow{\leftrightarrow} \\
-Z \overleftrightarrow{\mu}^{-1}(\vec{r}) \cdot \nabla \times & 0
\end{array}\right) \odot\left(\begin{array}{c}
\vec{E}(\vec{r}, t) \\
Z H(\vec{r}, t)
\end{array}\right)-\left(\begin{array}{c}
\stackrel{\leftrightarrow}{\varepsilon}^{-1}(\vec{r}) \cdot \vec{J} e(\vec{r}, t) \\
\stackrel{\leftrightarrow}{\mu}^{-1}(\vec{r}) \cdot \vec{J} h(\vec{r}, t)
\end{array}\right) \\
& \overleftrightarrow{0}=\left(0_{n, m}\right)=\left(\begin{array}{lll}
0 & 0 & 0 \\
0 & 0 & 0 \\
0 & 0 & 0
\end{array}\right)=\left(\begin{array}{l}
0 \\
0 \\
0
\end{array}\right)\left(\begin{array}{l}
0 \\
0 \\
0
\end{array}\right) \quad \text { (zero dyadic) }
\end{aligned}
$$

where $Z$ is a normalizing impedance which we can choose at our convenience. This is already in a form which looks like a product integral [13], except that the matrix to be integrated is a differential operator (with respect to space). Conductivities (electric and Magnetic) can also be included.

Attempting to find a combined-field form as in (2.7) we can write

$$
\nabla \times \vec{E}_{q}(\vec{r}, t)+\overleftrightarrow{\mu}(\vec{r}) \cdot \frac{\partial \vec{H}(\vec{r}, t)}{\partial t}-q j Z \stackrel{\leftrightarrow}{\varepsilon}(\vec{r}) \cdot \frac{\partial \vec{E}(\vec{r}, t)}{\partial t}=q j \vec{J}_{q}(\vec{r}, t)
$$

This does not combine as neatly as before. However, let us try to make this equation only involve $\vec{E}_{q}$ and $\vec{J}_{q}$. So we set

$$
\begin{aligned}
& \overleftrightarrow{\mu}(\vec{r}) \cdot \vec{H}(\vec{r}, t)-q j Z \overleftrightarrow{\varepsilon}(\vec{r}) \cdot \vec{E}(\vec{r}, t)=-q j Z \overleftrightarrow{\varepsilon}(\vec{r}) \cdot \vec{E} q(\vec{r}, t)
\end{aligned}
$$

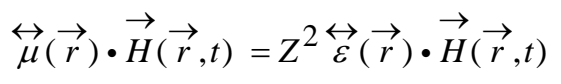

$$
\begin{aligned}
& \overleftrightarrow{\mu}(\vec{r}) \cdot \overleftrightarrow{\varepsilon}^{-1}(\vec{r})=\overleftrightarrow{\varepsilon}^{-1}(\vec{r}) \cdot \overleftrightarrow{\mu}(\vec{r})=Z^{2} \overleftrightarrow{1}
\end{aligned}
$$


This is an isoimpedance medium with wave impedance $Z$ which is position independent. This is contrasted to the usual isorefractive medium for which the product $\mu \varepsilon$ (or square of the refractive index) is position independent.

We can now write a first order (in time) $3 \times 3$ matrix differential equation as the combined Maxwell equation

$$
\left[\nabla \times-q j Z \stackrel{\leftrightarrow}{\varepsilon} \cdot \frac{\partial}{\partial t}\right] \vec{E}_{q}(\vec{r}, t)=q j Z \vec{J}_{q}(\vec{r}, t)
$$

In this special case of an isoimpedance medium the problem is reduced to $3 \times 3$ instead of $6 \times 6$. Let us remember this in the development that follows. At this point we can note that some cases of isoimpedance media $(\mu / \varepsilon$ independent of position) have been encountered in examples of transient lenses [15].

Rearranging (3.5) as

$$
\frac{\partial}{\partial t} \vec{E}_{q}(\vec{r}, t)=q j Z^{-1} \stackrel{\leftrightarrow}{\varepsilon}^{-1}(\vec{r}) \cdot \nabla \times \vec{E}_{q}(\vec{r}, t) \quad-\overleftrightarrow{\varepsilon}^{-1}(\vec{r}) \cdot \overleftrightarrow{J}_{q}(\vec{r}, t)
$$

we have a $3 \times 3$ first-order differential equation in $t$, analogous to the $6 \times 6$ form in (3.2). Both can be treated in the same general way. 
4. Propagating Electromagnetic Fields

For notational convenience we first have

$$
\begin{aligned}
& \nabla \times=\overleftrightarrow{\mathrm{C}} \text {. } \\
& \overleftrightarrow{\mathrm{C}}=\left(\begin{array}{ccc}
0 & -\frac{\partial}{\partial z} & \frac{\partial}{\partial y} \\
\frac{\partial}{\partial z} & 0 & -\frac{\partial}{\partial x} \\
-\frac{\partial}{\partial y} & \frac{\partial}{\partial x} & 0
\end{array}\right)=-\overleftrightarrow{\mathrm{C}} T \\
& Z^{-1 \overleftrightarrow{\varepsilon}^{-1}}(\vec{r}) \cdot \nabla \times=Z^{-1} \overleftrightarrow{\varepsilon}^{-1}(\vec{r}) \cdot \stackrel{\leftrightarrow}{\mathrm{C}} \cdot \\
& -Z \overleftrightarrow{\mu}^{-1}(\vec{r}) \cdot \nabla \times=-Z \overleftrightarrow{\mu}^{-1}(\vec{r}) \cdot \stackrel{\leftrightarrow}{\mathrm{C}}
\end{aligned}
$$

This lets us write our operators in dot product (contraction) form. For (3.2) we can then define

$$
\begin{aligned}
& \left(\begin{array}{cc}
\stackrel{\leftrightarrow}{\mathrm{O}} & Z^{-1 \overleftrightarrow{\varepsilon}^{-1}}(\vec{r}) \cdot \nabla \times \\
-Z \overleftrightarrow{\mu}^{-1}(\vec{r}) \cdot \nabla \times & \stackrel{\mathrm{O}}{(\vec{r})}
\end{array}\right) \odot=\left(\left(\Xi_{n, m}(\vec{r})\right)_{v, v^{\prime}}\right) \odot
\end{aligned}
$$

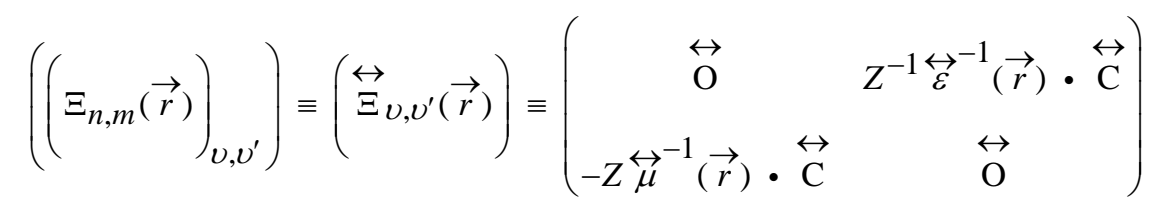

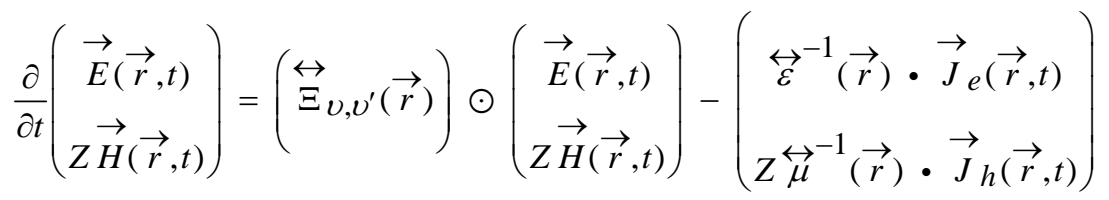

giving a compact form for the first-order differential equation in time. Similarly for the isoimpedance medium (3.6) becomes

$$
\begin{aligned}
& q j Z^{-1 \overleftrightarrow{\varepsilon}^{-1}}(\vec{r}) \cdot \nabla \times=\overleftrightarrow{\Xi}_{q}(\vec{r}) \cdot \\
& \stackrel{\leftrightarrow}{\Xi}_{q}(\vec{r})=q j Z^{-1 \overleftrightarrow{\varepsilon}^{-1}}(\vec{r}) \cdot \stackrel{\leftrightarrow}{\mathrm{C}} \\
& \frac{\partial}{\partial t} \vec{E}_{q}(\vec{r}, t)=\overleftrightarrow{\Xi}_{q}(\vec{r}) \cdot \vec{E}_{q}(\vec{r}, t)-\overleftrightarrow{\varepsilon}^{-1}(\vec{r}) \cdot \vec{J}_{q}(\vec{r}, t)
\end{aligned}
$$


Now (4.2) and (4.3) have the same form except that the first is a supermatrix $(6 \times 6)$ equation while the second is a matrix $(3 \times 3)$ equation.

As has been observed $[8,14]$, one can approach the path integral by finding a propagator (which is different from a Green function). For this purpose we have the general form of the homogeneous differential equation (no source currents) as

$$
\begin{gathered}
\frac{\partial}{\partial t}\left(P_{n, m}\left(\vec{r}, \overrightarrow{r^{\prime}} ; t, t^{\prime}\right)\right)=\left(Q_{n, m}(\vec{r})\right) \cdot\left(P_{n, m}\left(\vec{r}, \overrightarrow{r^{\prime}} ; t, t^{\prime}\right)\right) \\
\left(P_{n, m}\left(\vec{r}, \overrightarrow{r^{\prime}} ; t, t^{\prime}\right)\right) \equiv \text { propagator }
\end{gathered}
$$

The matrix operator $\left(Q_{n, m}(\vec{r})\right)$, can be interpreted as either the $6 \times 6$ form in (4.2), or the $3 \times 3$ form in (4.3). For the solution of (4.4) we first observe that

$$
\left(P_{n, m}\left(\vec{r}, \overrightarrow{r^{\prime}} ; t, t^{\prime}\right)\right)=e^{\left(Q_{n, m}(\vec{r})\right)\left[t-t^{\prime}\right]} \cdot\left(P_{n, m}^{(0)}\left(\vec{r}, \vec{r}^{\prime}\right)\right)
$$

By differentiating this with respect to $t$ we can see that (4.4) is reproduced.

To normalize this we enforce the identity for $\overrightarrow{r^{\prime}} \rightarrow \vec{r}, t^{\prime} \rightarrow t$ as

$$
\lim _{t^{\prime} \rightarrow t}\left(P_{n, m}\left(\vec{r}, \overrightarrow{r^{\prime}} ; t, t^{\prime}\right)\right)=\left(1_{n, m}\right) \delta\left(\vec{r}-\vec{r}^{\prime}\right)=\left(P_{n, m}^{(0)}\left(\vec{r}-\overrightarrow{r^{\prime}}\right)\right)
$$

giving

$$
\left(P_{n, m}\left(\vec{r}, \vec{r}^{\prime} ; t, t^{\prime}\right)\right)=e^{\left(Q_{n, m}(\vec{r})\right)\left[t-t^{\prime}\right]} \delta\left(\vec{r}-\vec{r}^{\prime}\right)
$$

as a solution for the propagator.

By taking a spatial Fourier transform of the delta function we have a representation as 


$$
\begin{aligned}
\delta\left(\vec{r}-\overrightarrow{r^{\prime}}\right) & =[2 \pi]^{-3} \int_{V_{\kappa}} e^{j \vec{\kappa} \cdot\left[\vec{r}-\overrightarrow{r^{\prime}}\right]} d V_{\kappa} \\
& =[2 \pi]^{-3} \int_{V_{\kappa}} e^{-j \vec{\kappa} \cdot\left[\vec{r}-\vec{r}^{\prime}\right]} d V_{\kappa} \\
\vec{\kappa} & \equiv \kappa_{x} \overrightarrow{1}_{x}+\kappa_{y} \overrightarrow{1}_{y}+\kappa_{z} \overrightarrow{1}_{z} \\
& \equiv \text { spatial frequency }
\end{aligned}
$$

The integral over $V_{\kappa}$ is over all possible real values of $\vec{\kappa}$, i.e., a triple integral over $-\infty$ to $+\infty$. In this form we have

$$
\left(P_{n, m}\left(\vec{r}, \overrightarrow{r^{\prime}} ; t-t^{\prime}\right)\right)=\int_{V_{\kappa}} e^{\left(Q_{n, m}(\vec{r})\right)\left[t-t^{\prime}\right]} e^{j \vec{\kappa} \cdot\left[\vec{r}-\vec{r}^{\prime}\right]} d V_{\kappa}
$$

While we may think that the exponentials can be combined since any matrix commutes with the identity matrix, the matrix operator involves spatial derivatives which do not commute with $\left(1_{n, m}\right) j \vec{\kappa} \cdot\left[\vec{r}-\overrightarrow{r^{\prime}}\right]$.

One can write out the power series as

$$
e^{\left(Q_{n, m}(\vec{r})\right)\left[t-t^{\prime}\right]}=\left(1_{n, m}\right)+\sum_{p=1}^{\infty} \frac{\left[t-t^{\prime}\right]^{p}}{p !}\left(Q_{n, m}(\vec{r})\right)^{p}
$$

Here $\left(Q_{n, m}(\vec{r})\right)^{p}$ is not just the $p$ th power, but a successive p-fold operation. Note that

$$
\begin{aligned}
& \frac{\partial}{\partial x} e^{j \vec{\kappa} \cdot\left[\vec{r}-\overrightarrow{r^{\prime}}\right]}=j \kappa_{x} e^{j \vec{\kappa} \cdot\left[\vec{r}-\overrightarrow{r^{\prime}}\right]} \\
& \frac{\partial}{\partial y} e^{j \vec{\kappa} \cdot\left[\vec{r}-\overrightarrow{r^{\prime}}\right]}=j \kappa_{y} e^{j \vec{\kappa} \cdot\left[\vec{r}-\overrightarrow{r^{\prime}}\right]} \\
& \frac{\partial}{\partial z} e^{j \vec{\kappa} \cdot\left[\vec{r}-\overrightarrow{r^{\prime}}\right]}=j \kappa_{z} e^{j \vec{\kappa} \cdot\left[\vec{r}-\overrightarrow{r^{\prime}}\right]}
\end{aligned}
$$

This gives for the $p=1$ term 


$$
\begin{aligned}
& {\left[t-t^{\prime}\right] Z^{-1} \stackrel{\leftrightarrow}{\varepsilon}(\vec{r}) \cdot \stackrel{\leftrightarrow}{\mathrm{C}} e^{j \vec{\kappa} \cdot\left[\vec{r}-\vec{r}^{\prime}\right]}=j\left[t-t^{\prime}\right] Z^{-1} \stackrel{\leftrightarrow}{\varepsilon}-1(\vec{r}) \cdot\left(\begin{array}{ccc}
0 & -\kappa_{z} & \kappa_{y} \\
\kappa_{z} & 0 & -\kappa_{X} \\
-\kappa_{y} & -\kappa_{X} & 0
\end{array}\right)} \\
& {\left[t-t^{\prime}\right] Z \overleftrightarrow{\mu}^{-1}(\vec{r}) \cdot \stackrel{\leftrightarrow}{\mathrm{C}} e^{j \vec{\kappa} \cdot\left[\vec{r}-\vec{r}^{\prime}\right]}=j\left[t-t^{\prime}\right] Z \overleftrightarrow{\mu}^{-1}(\vec{r}) \cdot\left(\begin{array}{ccc}
0 & -\kappa_{z} & \kappa_{y} \\
\kappa_{z} & 0 & -\kappa_{X} \\
-\kappa_{y} & -\kappa_{X} & 0
\end{array}\right)}
\end{aligned}
$$

For the $p=2$ term we now have to include $\stackrel{\leftrightarrow}{\varepsilon}^{-1}(\vec{r})$ and $\overleftrightarrow{\mu}^{-1}(\vec{r})$ in the spatial derivatives as well as $e^{j \vec{\kappa} \cdot\left[\vec{r}-\vec{r}^{\prime}\right]}$. Going to the general $p$ th term this becomes more and more complicated. While the representation is valid, actual computations become more and more tedious.

For the simpler case of a uniform isotropic medium we have for the $6 \times 6$ case

$$
\begin{aligned}
\overleftrightarrow{\varepsilon}(\vec{r}) & =\varepsilon \overleftrightarrow{1}, \overleftrightarrow{\mu}(\vec{r})=\mu \overleftrightarrow{1} \\
Z & =\left[\frac{\mu}{\varepsilon}\right]^{1 / 2}, \quad \mathrm{v}=[\mu \varepsilon]^{-1 / 2}
\end{aligned}
$$

which now reduces yo the $3 \times 3$ case as in (3.6) in the simpler form

$$
\begin{aligned}
\frac{\partial}{\partial t} \vec{E}_{q}(\vec{r}, t) & =\overleftrightarrow{\Xi}_{q}(\vec{r}) \cdot \vec{E}_{q}(\vec{r}, t)-\varepsilon^{-1} \vec{J}_{q}(\vec{r}) \\
\stackrel{\leftrightarrow}{\Xi}_{q}(\vec{r}) & =q j \mathrm{\leftrightarrow} \mathrm{C}
\end{aligned}
$$

In this case we have the propagator

$$
\begin{aligned}
& e^{\stackrel{\leftrightarrow}{\Xi}_{q}(\vec{r})\left[t-t^{\prime}\right]}=1+\sum_{p=1}^{\infty} \frac{\left[t-t^{\prime}\right]^{p}}{p !} \overleftrightarrow{\Xi}_{q}^{p}(\vec{r}) \\
& \stackrel{\leftrightarrow}{P}_{q}\left(\vec{r}, \overrightarrow{r^{\prime}} ; t-t^{\prime}\right)=\int_{V_{\kappa}} e^{\stackrel{\Xi}{\Xi}_{q}(\vec{r})\left[t-t^{\prime}\right]} e^{j \stackrel{\leftrightarrow}{\kappa} \cdot\left[\vec{r}-\vec{r}^{\prime}\right]} d V_{\kappa} \\
& \stackrel{\leftrightarrow}{\Xi}_{p}^{p}(\vec{r})=[j q v]^{p}\left(\begin{array}{ccc}
0 & -\kappa_{z} & \kappa_{y} \\
\kappa_{z} & 0 & -\kappa_{X} \\
-\kappa_{y} & \kappa_{X} & 0
\end{array}\right)^{p} \\
& \stackrel{\leftrightarrow}{P}_{q}\left(\vec{r}, \vec{r}^{\prime} ; t-t^{\prime}\right)=\int_{V_{\kappa}} e j\left(\begin{array}{ccc}
0 & -\kappa_{z} & \kappa_{y} \\
\kappa_{z} & 0 & -\kappa_{X} \\
-\kappa_{y} & \kappa_{X} & 0
\end{array}\right)\left[V_{\kappa}\right.
\end{aligned}
$$


In this case the exponential series sums in a simple form.

$$
\begin{aligned}
& \text { Letting }\left(F_{n, m}(\vec{r}, t)\right) \text { represent a 6- or 3-component field vector as in (4.2) or (4.3) we have } \\
& \left(F_{n, m}(\vec{r}, t)\right)=\int_{V_{r}}\left(P_{n, m}\left(\vec{r}, \vec{r}^{\prime} ; t, t^{\prime}\right)\right) \cdot\left(F_{n, m}\left(\vec{r}^{\prime}, t^{\prime}\right)\right) d V_{r}
\end{aligned}
$$

which is a volume integral, now over space. This is limited to regions without sources. By enforcing causality one can ensure that the integrals are not extended over regions with sources. 


\section{Green Function}

Rearrange the form of (4.2) and (4.3) as

$$
\left[\frac{\partial}{\partial t} \quad-\left(Q_{n, m}(\vec{r})\right) \cdot\right]\left(E_{n}\left(\overrightarrow{r^{\prime}}, t^{\prime}\right)\right)=\left(J_{n}(\vec{r}, t)\right)
$$

where ( $E_{n}$ ) represents the $6 \times 6$ or $3 \times 3$ form of the fields and similarly ( $J_{n}$ ) for the source currents. We have the result for the propagator as

$$
\begin{gathered}
{\left[\left(1_{n, m}\right) \frac{\partial}{\partial t}-\left(Q_{n, m}(\vec{r})\right)\right] \cdot\left(P_{n, m}\left(\vec{r}, \vec{r}^{\prime} ; t, t^{\prime}\right)\right)=\left(0_{n, m}\right)} \\
\left(P_{n, m}\left(\vec{r}, \overrightarrow{r^{\prime}} ; t, t^{\prime}\right)\right)=e^{\left(Q_{n, m}(\vec{r})\right)\left[t-t^{\prime}\right]} \delta\left(\vec{r}-\vec{r}^{\prime}\right)
\end{gathered}
$$

From this we can construct the Green function as $[9,14]$

$$
\left(G_{n, m}\left(\vec{r}, \overrightarrow{r^{\prime}} ; t, t^{\prime}\right)\right)=\left(P_{n, m}\left(\vec{r}, \overrightarrow{r^{\prime}} ; t, t^{\prime}\right)\right) u\left(t-t^{\prime}\right)
$$

which satisfies the equation

$$
\left[\left(1_{n, m}\right) \frac{\partial}{\partial t}-\left(Q_{n, m}(\vec{r})\right)\right] \cdot\left(G_{n, m}\left(\vec{r}, \vec{r}^{\prime} ; t, t^{\prime}\right)\right)=\left(1_{n, m}\right) \delta\left(\vec{r}-\vec{r}^{\prime}\right) \delta\left(t-t^{\prime}\right)
$$

Now we have the solution for the fields as

$$
\begin{aligned}
\left(E_{n}(\vec{r}, t)\right) & =\int_{V} \int_{t_{0}}^{t_{+}}\left(G\left(\vec{r}, \overrightarrow{r^{\prime}} ; t, t^{\prime}\right)\right) \cdot\left(J_{n}\left(\overrightarrow{r^{\prime}}, t^{\prime}\right)\right) d t^{\prime} d V^{\prime} \\
& +\int_{V}\left(G\left(\vec{r}, \overrightarrow{r^{\prime}} ; t, t^{\prime}\right)\right) \cdot\left(E\left(\overrightarrow{r^{\prime}}, t_{0}\right)\right) d V^{\prime}
\end{aligned}
$$

where boundary contributions are not included for present purposes. Here $t_{0}$ is some initial time for which we have specified the spatial distribution of the fields. This applies in both $6 \times 6$ and $3 \times 3$ cases by appropriate substitution of terms from (4.2) and (4.3). 
6. Interpretation as Path Integral

To construct a path integral from (5.5), let the region near $\overrightarrow{r^{\prime}}$ have no sources, so we can write

$$
\left(E_{n}(\vec{r}, t)\right)=\int_{V}\left(G\left(\vec{r}, \overrightarrow{r^{\prime}} ; t, t_{n}\right)\right) \cdot\left(E_{n}\left(\vec{r}^{\prime}, t_{n}\right)\right) d V^{\prime}
$$

Where $t_{n}$ is now our initial time for which the fields are specified. This is a time-evolution equation for the fields for $t>t_{n}$.

Let $t=t_{0}$, our initial time. In this case (6.1) reduces to an identify

$$
\left(E_{n}\left(\vec{r}, t_{0}\right)\right)=\left(E_{n}\left(\vec{r}, t_{0}\right)\right)
$$

Now consider some small time step $\Delta t$ and define

$$
t_{n}=n \Delta t+t_{0} \text { for } n=1,2, \ldots
$$

At time $t_{1}$ we look out in space away from $\vec{r}$ to $\overrightarrow{r^{\prime}}$ near $\vec{r}$ at a distance, given by the $\overleftrightarrow{\mu}$ and $\stackrel{\leftrightarrow}{\varepsilon}$ of the medium, from which the fields can propagate from $\overrightarrow{r^{\prime}}$ to $\vec{r}$.

As in Fig. 6.1A consider some N space points "centered" on $\vec{r}$ (noting possible different propagation speeds in the various directions from $\vec{r}$. While Fig. 6.1 is illustrated on a planar surface, note that these N points are distributed around $\vec{r}$ in three dimensions. These N points are used to approximate the integral over $\vec{r}$ (using some appropriate domain surrounding each $\overrightarrow{r^{\prime}}$ chosen). This gives a sum of $\mathrm{N}$ terms to calculate the fields at $\vec{r}$. Choose any one of these terms to travel from $\overrightarrow{r^{\prime}}=\vec{r}_{1}$ to $\vec{r}$. We have designated the path as $P_{1}$. There are of course $\mathrm{N}$ possible choices for $P_{1}$.

Now repeat the process. Take our initial time as $t_{2}$. Start from one of the $\overrightarrow{r^{\prime}}$ which we have taken as $\vec{r}_{1}$ and calculate the fields here. As in Fig. 6.1B again take a set of $\mathrm{N}$ points around $\vec{r}_{1}$ which are $\Delta t$ away. Consider 


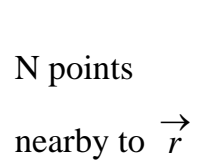

A. First time/space step in paths

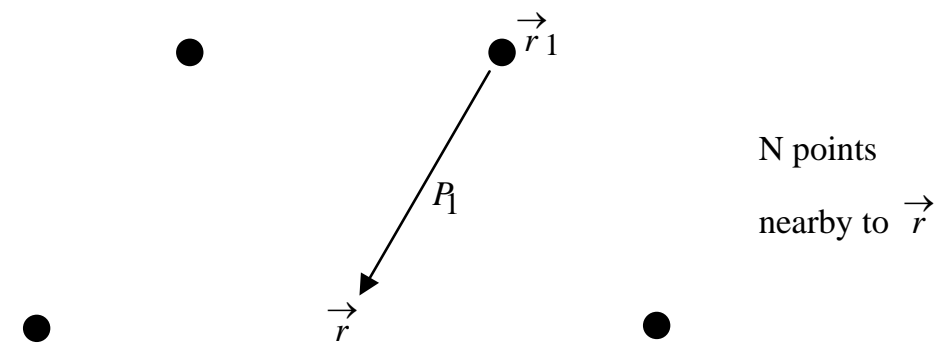

-

-

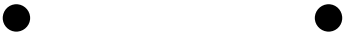


any one of these which we can call $\vec{r}_{2}$. The path from $\vec{r}_{2}$ to $\vec{r}_{1}$ can now be taken as part of $P_{1}$. (Note that $\vec{r}_{2}=\vec{r}$ is also one possible choice.) There are N possible choices leading to $\vec{r}_{1}$. With the previous result there are now $N^{2}$ possible choices for $P_{1}$.

Extending this by induction to an initial time $t_{n}$ we have $N^{n}$ possible choices for $P_{1}$. Increasing $\mathrm{N}$ to better approximate the integral, and taking $\Delta t$ smaller, the number of paths approaches $\infty$. This then is an appropriate interpretation of a path integral for the Maxwell equations. 


\section{Equivalence Principle}

Another well-known way to represent fields comes from the equivalence principle [1, 10]. Consider some volume $V_{e}$ bounded by a closed surface $S_{e}$, as in Fig. 7.1. Suppose that we have some known solution to the Maxwell equations valid in some large portion (or all)) of three-dimensional space as

$$
\vec{E}(\vec{r}, t) \text { and } \vec{H}(\vec{r}, t)
$$

There may be sources and the medium may be inhomogeneous/anisotropic.

The tangential fields on $S_{e}$ (coordinates $\vec{r}_{e}$ ) are given by

$$
\begin{aligned}
& \vec{E}_{t}\left(\vec{r}_{e}, t\right)=\overleftrightarrow{1}_{S}\left(\vec{r}_{e}\right) \cdot \vec{E}_{\left(\vec{r}_{e}, t\right)} \\
& \vec{H}_{t}\left(\vec{r}_{e}, t\right)=\overleftrightarrow{1}_{S}\left(\vec{r}_{e}\right) \cdot \vec{H}_{(}\left(\vec{r}_{e}, t\right) \\
& \overleftrightarrow{1}_{S}\left(\vec{r}_{e}\right)=\overleftrightarrow{1}-\overleftrightarrow{1}_{S}\left(\vec{r}_{e}\right) \overleftrightarrow{1}_{S}\left(\vec{r}_{e}\right) \\
& \overleftrightarrow{1}_{S}\left(\vec{r}_{e}\right) \equiv \text { outward pointing unit vector normal to } S_{e}
\end{aligned}
$$

If we can impose these as boundary conditions on $S_{e}$, then we can have the same fields as in (7.1) in $V_{e}$ as in (7.1) with no fields or sources outside, but any previous sources inside. Conversely we can remove fields and/or sources in $V_{e}$ and (7.2) will give the fields in (7.1) outside $V_{e}$ provided we retain any outside sources.

For this purpose we can construct equivalent electric $\vec{J}_{s_{e}}\left(\vec{r}_{e}\right)$ and magnetic $\vec{J}_{s_{h}}\left(\vec{r}_{e}\right)$ surface current density sources on $S_{e}$. The tangential components of the fields are discontinuous through the surface $\Sigma$ (Fig. 7.2) according to

$$
\begin{aligned}
& \vec{J}_{s_{e}}=\overrightarrow{1}_{\Sigma} \times\left[\vec{H}^{(+)}-\vec{H}^{(-)}\right] \\
& \vec{J}_{S_{h}}=-\overrightarrow{1}_{\Sigma} \times\left[\vec{E}^{(+)}-\vec{E}^{(-)}\right] \\
& \overrightarrow{1}_{\Sigma}=\text { unit vector normal to } \Sigma \text { pointing to + side }
\end{aligned}
$$




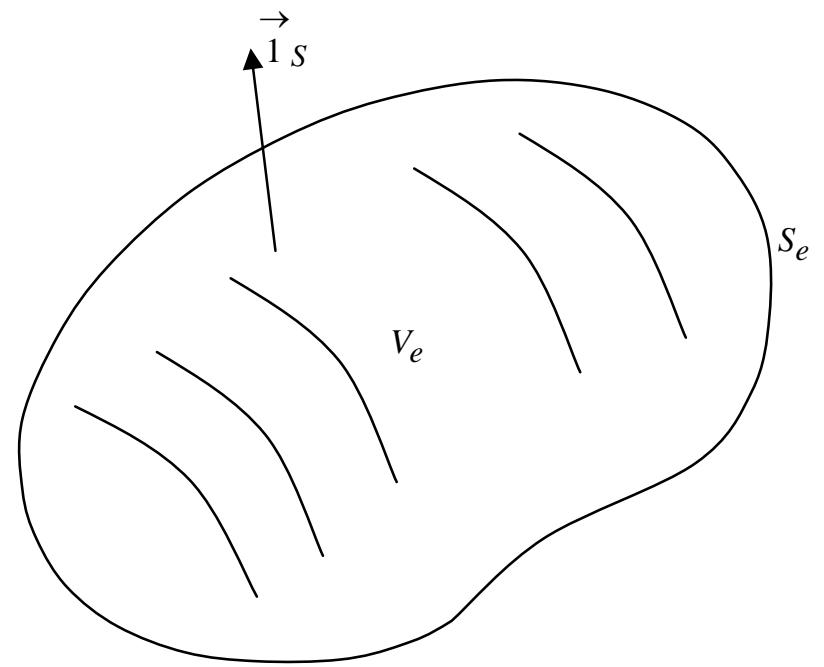

Fig. 7.1 Field Equivalence Principle for Constructing Fields Either Inside or Outside a Volume.

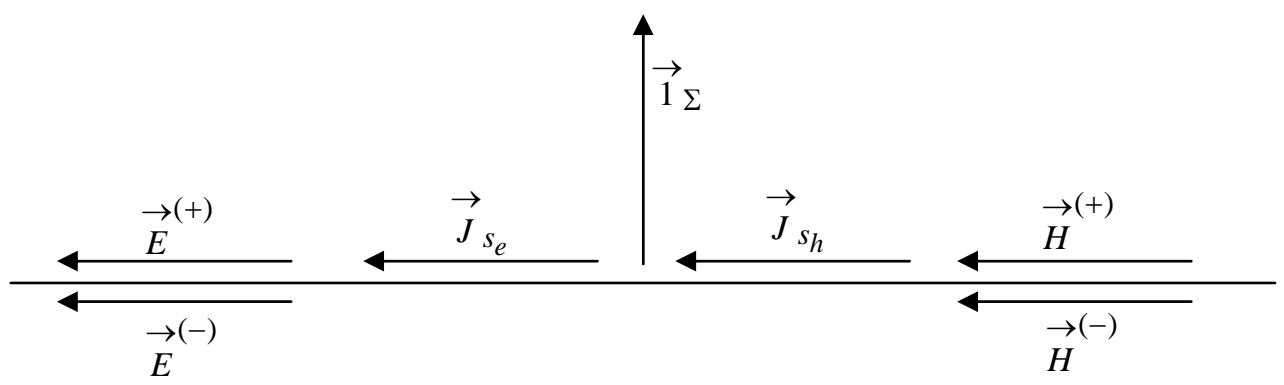

Fig. 7.2 Boundary Conditions 
So for sources in $V_{e}$ (inside $S_{e}$ ) we have

$$
\begin{aligned}
& \vec{J}_{s_{e}}\left(\vec{r}_{e}, t\right)=\overrightarrow{1}_{S}\left(\vec{r}_{e}\right) \times \vec{H}_{\left(\vec{r}_{e+}, t\right)} \\
& \vec{J}_{s_{h}}\left(\vec{r}_{e}, t\right)=-\overrightarrow{1}_{S}\left(\vec{r}_{e}\right) \times \vec{E}\left(\vec{r}_{e+, t}\right)
\end{aligned}
$$

Reproducing the external fields (with any exterior sources) with no inside fields or sources. Similarly for no fields or sources outside $S_{e}$ we have

$$
\begin{aligned}
& \vec{J}_{s_{e}}\left(\vec{r}_{e}, t\right)=-\overrightarrow{1}_{S}\left(\vec{r}_{e}, t\right) \times \vec{H}_{\left(\vec{r}_{e-}, t\right)} \\
& \vec{J}_{s_{h}}\left(\vec{r}_{e}, t\right)=\overrightarrow{1}_{S}\left(\vec{r}_{e}\right) \times \vec{E}\left(\vec{r}_{e-,}, t\right)
\end{aligned}
$$

for the external fields (including any exterior sources).

If the medium is uniform and isotropic (on the side of $S_{e}$ of interest), then the Green functions as in Section 2 can be used to integrate over these equivalent sources. Otherwise those in Section 5 can be used, provided one can calculate them, perhaps numerically.

Of course, one can apply the equivalence principle sequentially to a series of closed surfaces $S_{e}$, successive ones being contained inside the previous (perhaps for incoming waves). Alternately (perhaps for outgoing waves) successive $S_{e} s$ can enclose the previous. This gives a succession of surfaces, which might be considered as a one dimensional kind of path toward the field position of interest. Compare this to the more elaborate path structure represented by the path integral. 


\section{8. $\quad$ Concluding Remarks}

The intent of this paper has been to explore the path integral as an alternate representation of electromagnetic fields. Besides the Maxwell equations there are various integral representations. We have long been familiar with the use of Green functions to integrate over source currents to give the fields. One can also integrate with free-space Green functions over conduction currents to account for various media inhomogeneities/anisotropies.

The path integral provides a way to integrate over fields from previous times (initial conditions) to find the fields at later times. This can also include integrals over sources. As the initial time tends to $-\infty$, the usual Greenfunction integral over sources is left. As an added benefit we have found (Section 3) a special form of inhomogeneous/anisotropic medium for which the problem reduces from $6 \times 6$ matrices to $3 \times 3$ matrices.

These representation can also be compared to that from the field equivalence theorem. In this form the fields and sources in a volume can be replaced by equivalent electric and magnetic sheet currents on the boundary surface, or equivalently the tangential fields. While the path integral is based on initial conditions, the equivalence principle is based on boundary conditions.

Which representation one uses is a matter of convenience for the particular problem at hand. From a theoretical point of view, it is interesting that these various representations exist, all giving the correct result. This paper does not consider the relative advantages of the various representations for numerical calculations.

I would like to thank Robert Nevels for discussions concerning this subject. 
References

1. C. E. Baum, “The PARTES Concept in EMP Simulation”, Sensor and Simulation Note 260, December 1969.

2. B. K. Singaraju and C. E. Baum, "The Combined Field: Boundary Conditions, Integral Representation, Dyadic Green’s Functions and Some Theorems and Concepts”, Mathematics Note 38, September 1974.

3. C. E. Baum, “Application of Concepts of Advanced Mathematics and Physics to the Maxwell Equations”, Physics Note 11, November 1999; pp. 39-52, in P. D. Smith and S. R. Cloude (eds.), Ultra-Wideband, ShortPulse Electromagnetics 5, Kluwer Academic/Plenum Publishers, 2002.

4. C. E. Baum, "The Complementary Roles of Analysis, Synthesis, Numerics, and Experiment in Electromagnetics”, Physics Note 12, June 2001; ACES Journal, Vol. 20, No. 3, Nov. 2005, pp. 157-168.

5. C. E. Baum, “The Combined Field in Quaternion Form”, Physics Note 13, October 2003.

6. R. P. Feynman, “Space-Time Approach to Non-Relativistic Quantum Mechanics”, Rev. Modern Physics, Vol. 20, No. 2, April 1948, pp. 367-387.

7. C. E. Baum, "Emerging Technology for Transient and Broad-Band Analysis and Synthesis of Antennas and Scatterers”, Proc. IEEE, 1976, pp. 1598-1616.

8. R. D. Nevels, J. A. Miller, and R. E. Miller, "A Path Integral Time-Domain Method for Electromagnetic Scattering”, IEEE Trans. Antennas and Propagation, 2000, pp. 565-573.

9. R. D. Nevels and J. Jeong, “The Time Domain Green Function and Propagator for Maxwell's Equations”, IEEE Trans. Antennas and Propagation, 2004, pp. 3012-3018.

10. R. F. Harrington, Time-Harmonic Electromagnetic Fields, McGraw Hill, 1961.

11. R. P. Feynman and A. R. Hibbs, Quantum Mechanics and Path Integrals, McGraw Hill, 1965.

12. C. E. Baum, "The Singularity Expansion Method", ch. 3, pp. 129-179, in L. B. Felsen, Transient Electromagnetic Fields, Springer-Verlag, 1976.

13. J. D. Dollard and C. N. Friedman, Product Integration, Addison Wesley, 1979.

14. G. Barton, Elements of Green’s Functions and Propagation, Oxford U. Press, 1989.

15. C. E. Baum and A. P. Stone, Transient Lens Synthesis, Taylor \& Francis, 1991.

16. C. E. Baum and H. N. Kritikos, "Symmetry in Electromagnetics", ch. 1, pp. 1-90, in C. E. Baum and H. N. Kritikos (eds.), Electromagnetic Symmetry, Taylor \& Francis, 1995. 